\title{
Extended emission in Seyfert galaxies
}

\author{
Henrique A. Fraquelli ${ }^{1}$ and T. Storchi-Bergmann ${ }^{1}$ \\ ${ }^{1}$ Instituto de Física, UFRGS, C.P.15051, 91501-970, Porto Alegre, RS, Brazil \\ email: ico@if.ufrgs.br
}

\begin{abstract}
We use the photoionization code MAPPINGS Ic to model optical emission-line ratios of the extended emission-line regions of 24 Seyfert galaxies. Although most line ratios can be reproduced by varying the ionization parameter, gas density and/or the shape of the ionizing continuum, the ratio $[\mathrm{NII}] \lambda \lambda 6548,84 /[\mathrm{OII}] \lambda 3727$ can only be reproduced if we allow for a range of oxygen abundances of $0.5 \leqslant(\mathrm{O} / \mathrm{H}) /(\mathrm{O} / \mathrm{H})_{\odot} \leqslant 3$ for the whole sample. We propose a calibration to obtain $(\mathrm{O} / \mathrm{H})$ in terms of $[\mathrm{NII}] /[\mathrm{OII}]$. Also, in order to reproduce the emission-line spectra beyond $2 \mathrm{kpc}$ from the nucleus, we had to introduce the contribution from hot stars to the ionizing spectra. We conclude that this is due to an increasing contribution of HII regions with distance from the nucleus to the emission-line regions.
\end{abstract}

\section{Introduction}

The extended ionized gas is usually identified with the narrow-line region (NLR) of active galactic nuclei (AGN). The ENLR would correspond to the ionized gas beyond the NLR, where the gas velocities seem to be consistent with rotation within the galaxy (Peterson 1997). The ENLR emission could thus be attributed to interstellar gas at kiloparsec scales in the host galaxies ionized by the AGN nucleus. Photoionization by the nuclear source seems to be the main mechanism producing the observed emission (Morse, Raymond \& Wilson 1996; Evans et al. 1999). This conclusion is supported by self-consistent models applied to the ENLR (e.g. Fraquelli et al. 2000).

In this work, we investigate the chemical abundance of the extended gas, using the line ratio $[\mathrm{NII}] \lambda \lambda 6548,6584 /[\mathrm{OII}] \lambda 3727$, as previously done by Viegas \& Gouveia dal Pino (1992) and Viegas \& Prieto (1992), for this purpose. We also investigate, using photoionization modelling, the excitation properties of the extended gas, searching for signals of contributions from hot stars to the observed emission line ratios.

The sample comprises high signal-to-noise ratio long-slit spectra of 18 Seyfert 2 galaxies (Fraquelli et al. 2003) and 6 Seyfert 1 galaxies (Fraquelli 2002) that show ionized gas extending up to $4 \mathrm{kpc}$ from the nucleus. The slit width corresponded to $2^{\prime \prime}$ on the sky, with the slit oriented along the maximum extent of the high excitation gas observed in narrow-band images centered on [OIII] $\lambda 5007$ (Mulchaey, Wilson \& Tsvetanov 1996).

\section{Comparison between observations and $\mathbf{A}_{M / I}$ models}

From the comparison between model predictions and observations, we found that the data is best reproduced by a sequence of varying chemical abundance in the $\mathrm{A}_{M / I}$ photoionization scenario, with a power-law ionizing source (Binette et al. 1996). These are illustrated in the figures below. In Fig. 1a, solid lines represent different chemical abundances in the range $0.5 \leqslant(\mathrm{O} / \mathrm{H}) /(\mathrm{O} / \mathrm{H})_{\odot} \leqslant 3$. The power-law index is kept at $\alpha=-1.3$ and the gas density in the $\mathrm{MB}$ component $\mathrm{n}_{M B}=50 \mathrm{~cm}^{-3}$. Dashed lines correspond to the case in which $3 \%$ of the incident radiation can escape (while the solid lines correspond to no escape of the incident radiation). 

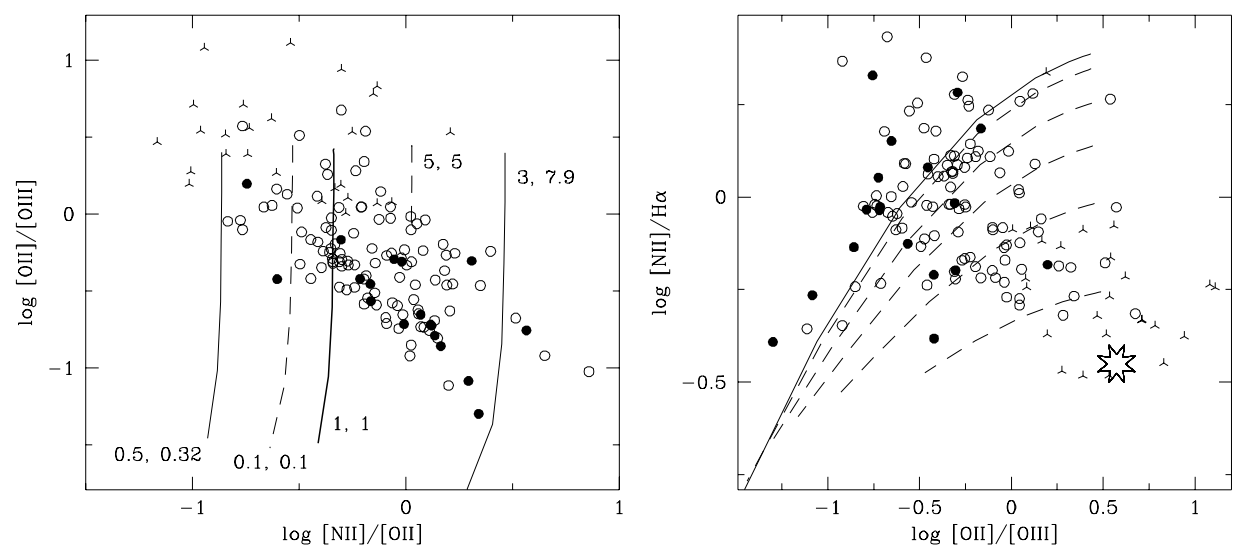

Figure 1. Left: $[\mathrm{OII}] /[\mathrm{OIII}]$ vs. $[\mathrm{NII}] /[\mathrm{OII}]$, for the data and $\mathrm{A}_{\mathrm{M} / \mathrm{I}}$ models, showing the difference in behaviour for primary Nitrogen (dashed lines) and secondary Nitrogen (solid lines). The labels by the lines are the corresponding Oxygen and Nitrogen abundances relative to the solar value, separated by a coma. Right: $[\mathrm{NII}] / \mathrm{H} \alpha$ vs. [OII]/[OIII], for the data and composite models, adopting secondary Nitrogen in models used previously, but assuming pure AGN ionizing continuum (solid lines), composite models (AGN + hot stars) containing an increasing contribution of hot stars to the ionizing continuum (dashed lines, from bottom to top), and pure hot star continuum (big star symbol). In both: different symbols represent three distance ranges: filled-circles for nuclei, open circles for $0.4 \leqslant \mathrm{r} \leqslant 2 \mathrm{kpc}$ and stars for $\mathrm{r}>2 \mathrm{kpc}$ from the nuclei.

We also investigate the possibility that the gas is ionized by both an AGN continuum and hot stars, in a composite model. Thus, we generate photoionization models where the ionization source is stars with $35000 \mathrm{~K}$, with mean gas density of $50 \mathrm{~cm}^{-3}$. These models are combined with the $\mathrm{A}_{M / I}$ models in different proportions of ionized gas. Fig. $1 \mathrm{~b}$ show these composite models compared to the data.

\section{Conclusions}

- At the nucleus and up to $2 \mathrm{kpc}$ from it, the excitation is similar and can be reproduced by models with a power-law ionizing continuum.

- The ratio $[\mathrm{NII}] \lambda \lambda 6548,84 /[\mathrm{OII}] \lambda 3727$ can only be reproduced if we allow for a range in oxygen abundance $0.5 \leqslant(\mathrm{O} / \mathrm{H}) /(\mathrm{O} / \mathrm{H})_{\odot} \leqslant 3$, adopting a secondary origin for Nitrogen.

- In order to reproduce the emission-line spectra beyond $2 \mathrm{kpc}$ from the nucleus, we had to introduce the contribution from hot stars to the ionizing spectrum. We conclude that this is due to an increasing contribution of HII regions with distance from the nucleus to the extended line emission.

\section{References}

Binette, L., Wilson, A. S., \& Storchi-Bergmann, T. 1996, A\&A, 312, 365

Evans, I. N., et al. 1997, ApJ, 521, 531

Fraquelli, H. A. 2002, PhD Thesis UFRGS.

Fraquelli, H. A., Storchi-Bergmann, T., \& Binette, L. 2000, ApJ, 532, 867

Fraquelli, H. A., Storchi-Bergmann, T., \& Levenson, N. A. 2003, MNRAS, 341, 449

Morse, J. A., Raymond, J. C., \& Wilson, A. S. 1996, PASP, 108, 426

Mulchaey, J., Wilson, A. S., \& Tsvetanov, Z. 1996, ApJS, 102, 309

Peterson, B. M. 1997, An introduction to active galactic nuclei (Cambridge University Press)

Viegas, S. M., \& Gouveia dal Pino, E. M. 1992, ApJ, 384, 467

Viegas, S. M., \& Prieto, M. A. 1992, MNRAS, 258, 483 